\title{
Conservative Management of Coloperitoneal-Vaginal Fistula
}

\author{
Antonia P. Francis, MD, Radu Apostol, DO, Ana Mrkaic, MD, Tara Berman, MD, Ido Sirota, MD, \\ Farr Nezhat, MD \\ Mount Sinai-Roosevelt Hospital, New York, NY, USA.
}

\begin{abstract}
Introduction: The occurrence of pelvic organ fistulas, after extensive pelvic surgery, has been widely described in the literature, as well as their surgical management via abdominal, vaginal, and laparoscopic approaches. Treatment depends on their etiology and location, as well as the surgeon's experience.

Case Description: We present the case of a coloperitoneal-colovaginal fistula, a unique clinical scenario. This developed as a complication of robotic-assisted laparoscopic resection of a pelvic mass, treatment of endometriosis, and trachelectomy. A conservative approach was used to treat the patient via interventional radiology-assisted drainage.
\end{abstract}

Discussion: Successful conservative management of a complex fistula between the colon, peritoneum, and vagina is feasible.

Key Words: Coloperitoneal fistula, Colovaginal fistula, Conservative management, Interventional radiology-assisted drainage.

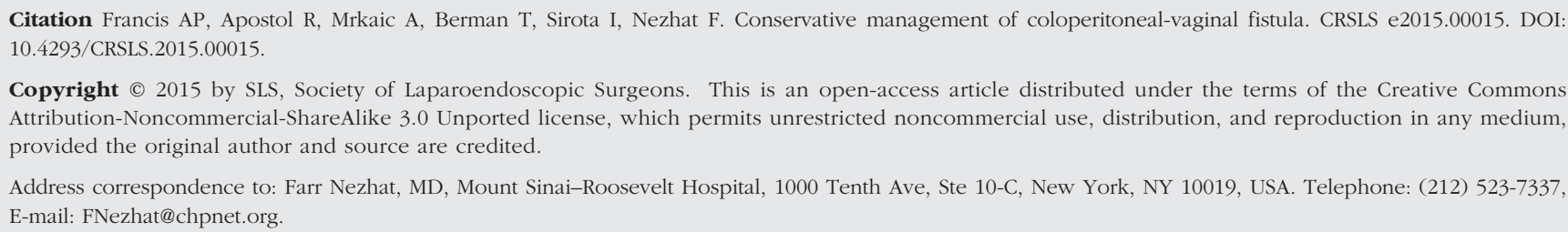

Copyright $\odot 2015$ by SLS, Society of Laparoendoscopic Surgeons. This is an open-access article distributed under the terms of the Creative Commons Attribution-Noncommercial-ShareAlike 3.0 Unported license, which permits unrestricted noncommercial use, distribution, and reproduction in any medium, provided the original author and source are credited.

Address correspondence to: Farr Nezhat, MD, Mount Sinai-Roosevelt Hospital, 1000 Tenth Ave, Ste 10-C, New York, NY 10019, USA. Telephone: (212) 523-7337, E-mail: FNezhat@chpnet.org.

\section{INTRODUCTION}

As a result of advances in modern obstetric care, fistulas are rare in the United States and other industrialized countries. However, iatrogenic injuries to the gastrointestinal or urinary tract during pelvic surgery, as well as postoperative infections, are the main risk factors contributing to fistula formation after challenging gynecologic cases.

Spontaneous fistula between the anorectum and vagina is extremely uncommon, comprising 5\% of all anorectal fistulas. ${ }^{1}$ Rectovaginal fistulas (RVFs) are abnormal epitheliumlined communications between the vagina and rectum. ${ }^{2}$ Moreover, the development of a coloperitoneal-colovaginal fistula is even more rare and presents a truly unique clinical scenario. The fistula wall is unlikely to spontaneously heal because of the increased intra-abdominal pressure in the abdomen from leakage of peritoneal fluid. Structurally, the anovaginal septum is thin and poorly vascularized. ${ }^{3}$ In addition, the inherently high pressures of the anorectal region continuously force fecal content through the fistula, while increased intra-abdominal pressure from the peritoneal fluid interferes with spontaneous healing. ${ }^{4}$ Other factors limiting the success of fistula healing include the etiology of the fistula; viability of surrounding tissues; patient comorbidities; and presence of inflammation, infection, and scar tissue. ${ }^{5}$ Treatment thus requires a methodical approach in choosing the appropriate modality to ensure success.

We report a successfully managed high, complex, coloperitoneal-colovaginal fistula by a conservative approach. At our institution, a case report is exempt from ethical approval by the institutional review board.

\section{CASE REPORT}

A 45-year-old woman, gravida II para I, with a long history of endometriosis and chronic pelvic pain, pre- 
sented with a recently diagnosed left complex adnexal mass $(6 \mathrm{~cm}$ in its largest diameter) and worsening of pelvic pain. Her medical history was also significant for prior supracervical hysterectomy and right salpingooophorectomy for symptomatic uterine leiomyoma, severe pelvic endometriosis, hypertension, diabetes mellitus, and peripheral neuropathy. She had been treated with a long duration of hormone suppressive therapy in the form of medroxyprogesterone acetate for pelvic pain and endometriosis; however, her symptoms persisted and she opted for extirpative surgical management. She underwent a robotic-assisted laparoscopic left salpingo-oophorectomy, resection of a pelvic mass, trachelectomy, extensive adhesiolysis, treatment of endometriosis, enterolysis, and primary repair of an intraoperative sigmoid enterotomy. Intraoperatively, the mass, confirmed on final pathologic analysis as endometrioma, was found severely attached to the bowel. Primary closure of the $1.5-\mathrm{cm}$ enterotomy was performed with simple interrupted No. 0 Vicryl sutures (Ethicon, Somerville, New Jersey) in 2 layers. Flexible sigmoidoscopy was performed to approximately $30 \mathrm{~cm}$, and the lumen of the sigmoid colon was completely intact and normal in appearance. The colon was then insufflated with air while immersed under saline solution, with no evidence of a leak from the repair.

Postoperatively, the patient underwent a workup for pulmonary emboli and received $2 \mathrm{U}$ of packet red blood cells for symptomatic anemia. She was discharged home on postoperative day (POD) 6, when she was able to tolerate a regular diet and had regained regular bowel function.

On POD 8, the patient presented with complaints of dark, foul-smelling vaginal discharge; nausea; worsening abdominal pain; and leukocytosis with a left shift. She was afebrile with no signs of peritonitis. Pelvic examination showed an opening of approximately $1 \mathrm{~cm}$ at the left vaginal apex, with drainage of stool. The patient was admitted with a presumptive diagnosis of RVF. Abdominopelvic computed tomography showed a large pelvic collection measuring $8.3 \times 7.3 \mathrm{~cm}$, suspicious for an abscess in the mid to left lower pelvis, localized to the site of the prior enterotomy repair (Figure 1). Delayed images were obtained to assess fistulous connection between the abscess and colon; however, no significant progress of contrast was observed. A 24F UreSil (LLC, Skokie, Illinois) drainage catheter was placed by an interventional radiologist, via a left anterolateral pelvic approach, to drain the fluid collection, which remained in place after the procedure. The drained material was feculent with yellow-

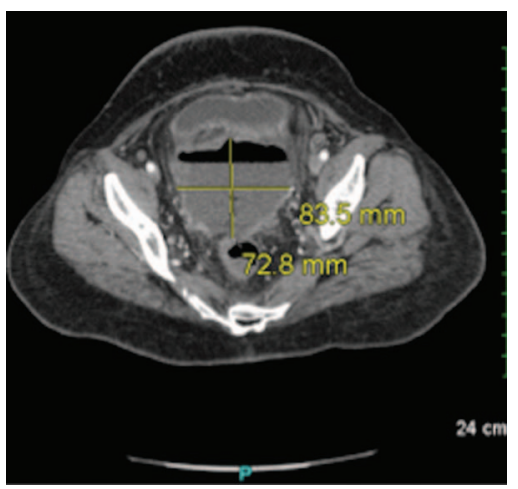

Figure 1. Abdominopelvic computed tomography scan showing a large pelvic collection measuring $8.3 \times 7.3 \mathrm{~cm}$.

brown stool draining at 50 to $300 \mathrm{~mL} / \mathrm{d}$. Empiric antibiotic and antifungal therapy was initiated with intravenous imipenem-cilastatin (Primaxin; Merck, Kenilworth, NJ), vancomycin, and fluconazole therapy and maintained after drain placement after pelvic abscess cultures showed a polymicrobial infection.

Throughout the hospital course, the patient responded appropriately to medical management with nothing by mouth, total parenteral nutrition, and continuation of the antibiotics administered through the peripherally inserted central catheter line. Two weeks after the procedure, repeat abdominopelvic computed tomography showed almost complete resolution of the collection with residual inflammatory changes surrounding the sigmoid colon (Figure 2). Contrast was evident in both the drainage catheter and extraluminal, within the abdominal cavity and vagina, leading to the diagnosis of peritoneal RVF. The patient remained afebrile, and pain steadily subsided, with decreasing drainage through both the intra-abdominal catheter and vagina. She was discharged home on hospital day 15 , receiving total parenteral nutrition, intravenous ertapenem (Invanz;

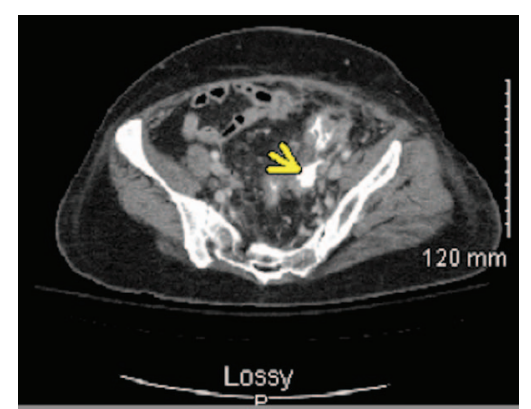

Figure 2. Abdominopelvic computed tomography scan showing complete resolution of collection. Arrow pointing to extraluminal contrast. 
Merck), and oral fluconazole. She was closely followed up regularly on an outpatient basis. The abdominal drain remained in place for an additional 32 days and was removed on POD 53. The patient was tolerating a regular diet, and on each follow-up visit, she showed signs of clinical improvement, with no further vaginal drainage and gradual closure of the vaginal opening. She continued to do well without any further complications 8 months after drainage.

\section{DISCUSSION}

There are multiple classifications of RVFs: high versus low and simple versus complex. Low versus high fistulas are classified based on the location in relation to the cervix and vaginal fourchette. ${ }^{6-8}$ Another classification is simple versus complex. Simple RVFs are defined as RVFs located in the middle or lower portion of the vaginal septum, $<2.5 \mathrm{~cm}$ in diameter, and caused by local trauma or sepsis. Complex fistulas are $>2.5 \mathrm{~cm}$ in diameter and located in the upper portion of the rectovaginal septum.

Currently, no evidence-based randomized controlled trials exist regarding the appropriate management of RVFs. ${ }^{6,7}$ Of the few cases of peritoneal fistulas reported, as well as RVFs, most have been repaired surgically. 4,9,10 Successful treatment usually involves surgical repair and depends on etiology and location, as well as the expertise of the surgeon. To our knowledge, spontaneous healing of only a simple RVF, developing after laparoscopic segmental resection for intestinal deep infiltrating endometriosis, has been reported. ${ }^{11}$

In high RVFs, the preferred transabdominal approach is often cited, with repair involving interposition of healthy tissue between the rectum and vagina with surgical modalities including low anterior resections, abdominal perineal resections, and complex muscle flaps. Successful laparoscopic treatment of mid and high RVFs has been reported. ${ }^{12,13}$ The perineal approach is often preferred for low fistulas. Less invasive procedures include bioprosthetic fistula plugs made from porcine intestinal submucosa, as well as fibrin or BioGlue Surgical Adhesive (CryoLife, Inc., Kennesaw, Georgia). Originally designed for anal fistulas, the plugs are now increasingly used for RVFs and can be applied in a transvaginal or transanal manner. ${ }^{14-16}$

To our knowledge, this is the first report of successful conservative management of a complex fistula involving a fistulous tract between the colon, peritoneum, and vagina. Only a small number of patients with RVFs respond to medical optimization. Most women have persistent symptomatic disease that will not heal without surgical intervention. ${ }^{8}$ Following the general recommendation to perform medical treatment in the face of inflammation or infection for 3 to 6 months, we postponed surgical management because our patient presented with an infected abdominal collection. ${ }^{8}$ When surgical therapy is not possible, fistulas should be treated with fecal diversion or an endoluminal stent. Therefore we adapted this less invasive, low-risk approach, consisting of drainage catheter placement and intravenous antibiotic and antifungal treatment. Using this approach, many fistulas will spontaneously close. Our patient had a high, complex fistula, which would have required a transabdominal surgical repair; however, with conservative treatment only, we were able to successfully treat the fistula. Our case can greatly contribute to the literature concerning this unique clinical scenario.

\section{References:}

1. Cera SM, Nogueras JJ. Rectovaginal Fistula. In: Davila GW, Ghoniem GM, Wexner SD, eds. Pelvic Floor Dysfunction. New York, NY: Springer; 2009:325-333.

2. Brown HW, Wang L, Bunker CH, Lowder JL. Lower reproductive tract fistula repairs in inpatient US women, 1979-2006. Int Urogynecol J. 2012;23(4):403-410.

3. Nassar OA. Primary repair of rectovaginal fistulas complicating pelvic surgery by gracilis myocutaneous flap. Gynecol Oncol. 2011;121:610-614.

4. Neumann JL, Moran J. Peritonitis due to a peritoneal vaginal fistula. Nephrol Nurs J. 2010;37(2):177-178, 181.

5. Pinto RA, Peterson TV, Shawki S, Davila GW, Wexner SD. Are there predictors of outcome following rectovaginal fistula repair? Dis Colon Rectum. 2010;53:1240-1247.

6. Zhu YF, Tao GQ, Zhou N, Xiang C. Current treatment of rectovaginal fistula in Crohn's disease. World J Gastroenterol. 2011;17(8):963-967.

7. Champagne BJ, McGee MF. Rectovaginal fistula. Surg Clin North Am. 2010;90(1):69-82.

8. Debeche-Adams TH, Bohl JL. Rectovaginal fistulas. Clin Colon Rectal Surg. 2010;23(2):99-103.

9. Jacobson MT, Osias J, Velasco A, Charles R, Nezhat C. Laparoscopic repair of a uteroperitoneal fistula. JSLS. 2003; 7(4):367-369. 
10. Nezhat CH, Bastidas JA, Pennington E, Nezhat FR, Raga F, Nezhat CR. Laparoscopic treatment of type IV rectovaginal fistula. J Am Assoc Gynecol Laparosc. 1998;5(3):297-299.

11. Kondo W, Ribeiro R, Trippia CH, Zomer MT. Spontaneous healing of a rectovaginal fistula developing after laparoscopic segmental bowel resection for intestinal deep infiltrating endometriosis. Case Rep Obstet Gynecol. 2013;2013:837903.

12. Schwenk W, Bohm B, Grundel K, Muller J. Laparoscopic resection of high rectovaginal fistula with intracorporeal colorectal anastomosis and omentoplasty. Surg Endosc. 1997;11:147-149.

13. Kumaran SS, Palanivelu C, Kavalakat AJ, Parthasarathi R, Neelayathatchi M. Laparoscopic repair of high rectovaginal fistula: is it technically feasible? BMC Surg. 2005;5:20.
14. Ellis CN. Outcomes after repair of rectovaginal fistulas using bioprosthetics. Dis Colon Rectum. 2008;1084(51):10841088.

15. Garcia S, Dissanaike S. Case report: treatment of rectovaginal fistula with Bioglue. Int J Surg Case Rep. 2012;3(7): 327-329.

16. De la Portilla F, Rada R, León E, Cisneros N, Maldonado VH, Espinosa E. Evaluation of the use of BioGlue in the treatment of high anal fistulas: preliminary results of a pilot study. Dis Colon Rectum. 2007;50(2):218-222. 